\title{
ON THE POSSIBILITIES OF PERMITTIVITY CALCULATION IN A CERTAIN BANDWIDTH FROM SINGLE FREQUENCY RESULTS
}

\author{
Antonio José Lozano Guerrero ${ }^{1}$, Juan Monzó-Cabrera ${ }^{1}$, Alejandro Díaz-Morcillo ${ }^{1}$ \\ ${ }^{1}$ Departamento de Tecnologías de la Información y las Comunicaciones, Universidad \\ Politécnica de Cartagena, Cartagena (Murcia) Spain 30202. \\ antonio.lozano@upct.es
}

Keywords: inverse technique, microwave, permittivity

\section{Introduction}

The permittivity of a material can be obtained from resonant measurements in an accurate way [1] at a single frequency (where the resonance occurs). In this work we propose and explore the possibilities of obtaining the permittivity of materials from resonant measurements in a certain frequency bandwidth around the resonance frequency. With this purpose a Debye model useful for polar liquids [1] jointly with a certain conductivity, are studied to evaluate this possibility jointly with inverse techniques.

The dielectric permittivity is a complex number

$$
\varepsilon^{*}=\varepsilon^{\prime}-j \varepsilon^{\prime \prime}
$$

and its dependence with the frequency can be obtained from [2] for polar liquids

$$
\varepsilon^{*}(\omega)=\varepsilon_{\infty}+\frac{\varepsilon_{S}-\varepsilon_{\infty}}{1+j \omega \tau}
$$

where: $\varepsilon^{\prime}$ is the dielectric constant, $\varepsilon^{\prime \prime}$ is the loss factor, $f$ is the frequency $(\mathrm{Hz}), \varepsilon_{S}$ and $\varepsilon_{\infty}$ are the dielectric constants at DC (low frequency) and at very high frequency, respectively. $\omega=2 \pi f(\mathrm{rad} / \mathrm{s})$ is the angular frequency. $\mathrm{T}(\mathrm{s})$ is the relaxation time of the system. It is defined as the time it takes the polarization of the material falling to 36.79 $\%$ of its value when the electric field is no longer applied.

To include the conductivity $\sigma(\mathrm{S} / \mathrm{m})$ in the Debye model [2] we have added to the permittivity model the term [3]

$$
\frac{\sigma}{\mathrm{j} \omega \varepsilon_{0}}
$$

where $\varepsilon_{0}=8.8542 \times 10^{-12}(\mathrm{~F} / \mathrm{m})$ is the free space permittivity and $j$ is the imaginary constant.

To include the temperature in the fitting models for expressions (1-2) we have used from [1] the following linear expressions for $\varepsilon_{s}$ and $\varepsilon_{\infty}$, and the exponential one for relaxation time T:

$$
\begin{aligned}
& \varepsilon_{\mathrm{s}}=\mathrm{a} \cdot \mathrm{T}+\mathrm{b} \\
& \varepsilon_{\infty}=c \cdot T+d
\end{aligned}
$$




$$
\tau=\tau_{0} \mathrm{e}^{\mathrm{E} / \mathrm{T}}
$$

where $T$ is the temperature and $E$ the activation energy. Coefficients $\left(a, b, c, d, \tau_{0}, E\right)$ were obtained by minimizing the fitness function (7).

$$
\text { feval }=\sqrt{\frac{\sum_{\mathrm{i}=1}^{\mathrm{N}}\left|\varepsilon_{\text {measured }}^{*}(\mathrm{i})-\varepsilon_{\text {model l ed }}^{*}(\mathrm{i})\right|^{2}}{\mathrm{~N}}}
$$

$\varepsilon_{\text {measured }}^{*}(i)$ is each frequency point for which the permittivity has been measured and $\varepsilon_{\text {model l ed }}^{*}(i)$ is each frequency point for which the permittivity has been modelled. $N$ is the number of non-equally temperature spaced samples which in our case is 7 .

\section{Method}

Samples were heated in a commercial microwave oven to near its boiling temperature. Its dielectric properties were measured at a frequency near $2 \mathrm{GHz}$ with a dielectrometer, which uses vials, ITACA (ITACA Dielkitv, Dielectrometer for vials, DIMAS, Spain.). Obtained data were processed by using Matlab (Mathworks, Natick, USA). As can be seen in Figure 1 an optical fiber from (Neoptix, Nomad Touch, Canada) was used to obtain the temperature. Measurements were acquired for temperature, frequency and permittivity while the temperature decreased. Various measurement rounds were carried out in order to test the repeatability of the procedure. Since permittivity changed with temperature resonant frequency also did as expected.

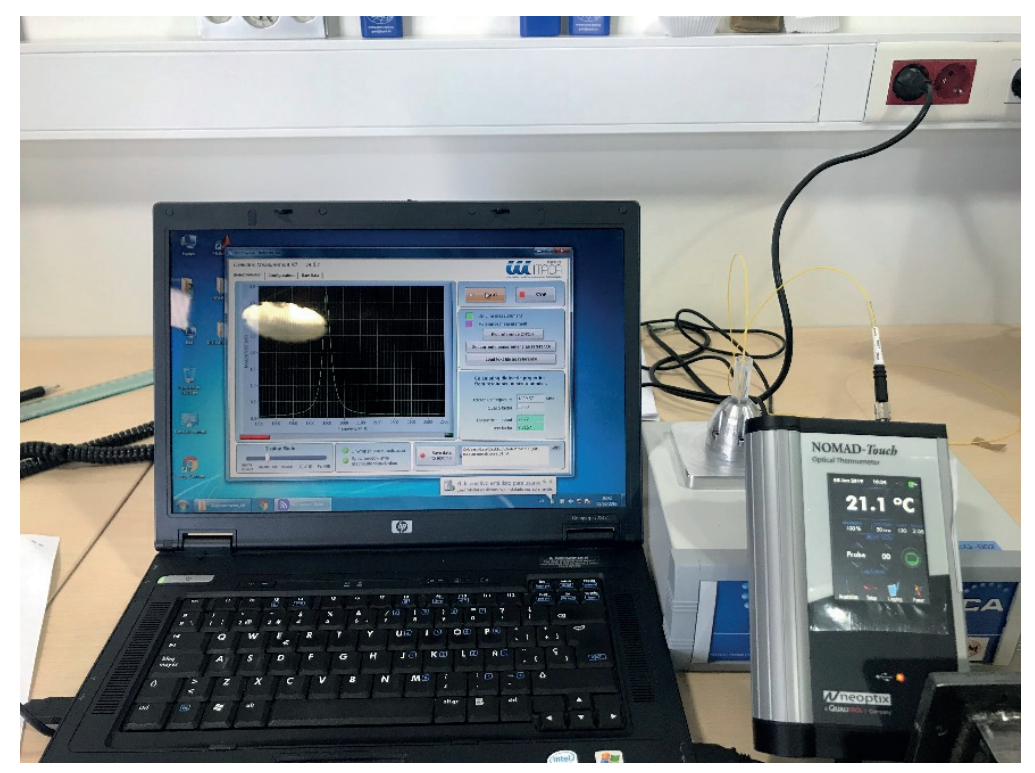

Fig. 1. Set up for the dielectrrometer with a fiber optic temperature probe. 
Concerning the optimization technique genetic algorithms are a powerful and wellestablished tool that has been used for the past 20 years successfully when a global optimum needs to be found. Extracting inversely the permittivity of materials at microwave frequencies has been studied in [4]. The parameters to be optimized depend on the unknowns for the Debye model including the term for the conductivity at the different temperatures. Upper and lower bound limits were established around the distilled water values except for the conductivity. To obtain the fitted models around 100 generations and a population of about 5000 were used in the studies. Genetic Algorithms jointly with gradient descend method have been used in Matlab (Mathworks, Natick, USA). Since most of the materials provide limited $\varepsilon_{\infty}$, this parameter can be fixed to a finite value. For instance: 1 , to reduce uncertainty and an excessive degree of freedom which may lead to wrong solutions.

\section{Results}

Optimized parameters are shown in Table I for distilled water provided in [1] and the ones obtained in this study. Jointly, the conductivity at each of the temperatures $(296.5,303$, $313.3,322.3,333.5,343.4$ and $357.3 \mathrm{~K}$ ) was also optimized. As we can see they do not differ much from results in [1] and they have been adjusted to our own measurements using only results from the available measurements.

Table 1. Parameter values for the Debye model for distilled water.

\begin{tabular}{ccccccc}
\hline Parameters & $\boldsymbol{a}$ & $\boldsymbol{b}$ & $\boldsymbol{c}$ & $\boldsymbol{d}$ & $\tau_{0}(\mathrm{~s})$ & $\boldsymbol{E}$ \\
\hline From [1] & -0.35 & 183.68 & -0.03 & 14.40 & $1.61 \mathrm{e}-15$ & 2470 \\
This work & -0.27 & 155.15 & 0 & 1 & $3.46 \mathrm{e}-15$ & 2278 \\
\hline
\end{tabular}

In Figure 2 and Figure 3, the dielectric constant and the loss factor of distilled water are shown respectively at different temperatures $(296.5,303,313.3,322.3,333.5,343.4$ and $357.3 \mathrm{~K})$. It can be observed how the dielectric constant and the loss factor generally decrease with temperature at the measurement frequencies. To fit the results a Debye model (2) including the ionic conductivity from (3) has been used. Expressions from (4-6) have been included and coefficients for $\left(a, b, c, d, \tau_{0}, E\right)$ were the parameters to be optimized jointly with the conductivity at each of the temperatures enforcing $\varepsilon_{\infty}=1$.

The fitting model results are represented with the discontinuous line in Figures 4 and 5 from $1.5 \mathrm{GHz}$ to $100 \mathrm{GHz}$ and reproduce the permittivity values better at frequencies close to the measurement ones. For these frequencies several values for $\varepsilon_{\mathrm{s}}, \varepsilon_{\infty}$ and $\mathrm{T}$ may provide reasonable optimums for (7) provided that the measured frequency range is limited to $1905 \mathrm{MHz}$ to $1950 \mathrm{MHz}$ and taking into account that our measurements show a noisy behavior. For this reason, upper and lower bounds for the relaxation time parameters have been adjusted around the theoretical ones to provide reasonable results. For the optimized conductivity $(296.5,303,313.3,322.3,333.5,343.4$ and $357.3 \mathrm{~K})$ results show a good repeatability and can be observed in Figure 6 . Values near the expected $0 \mathrm{~S} / \mathrm{m}$ for the distilled water are obtained. 


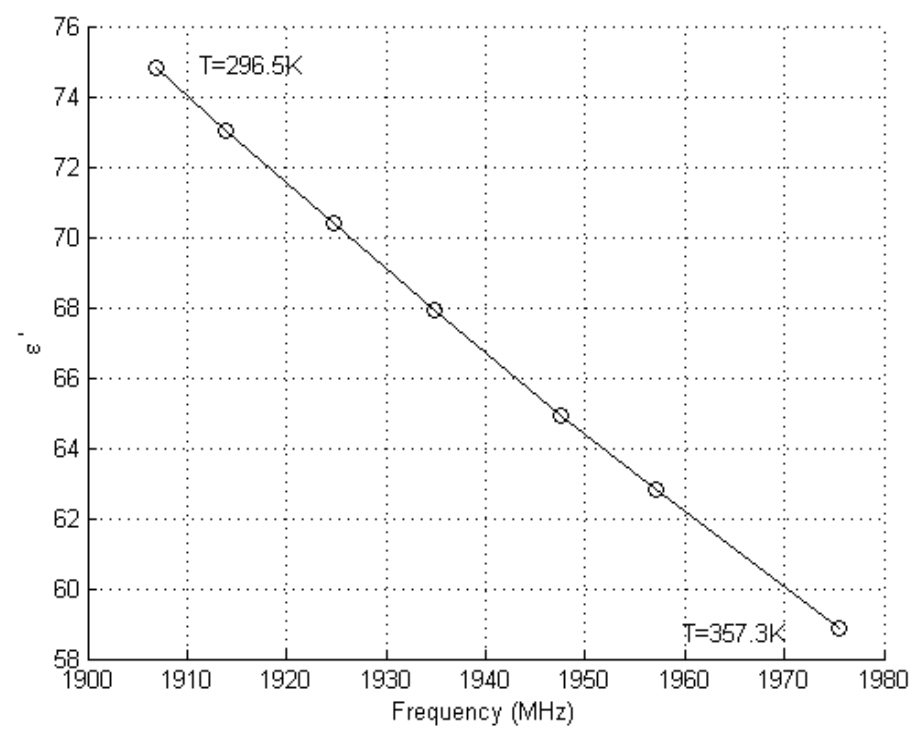

Fig. 2. Measured dielectric constant of distilled water at different temperatures.

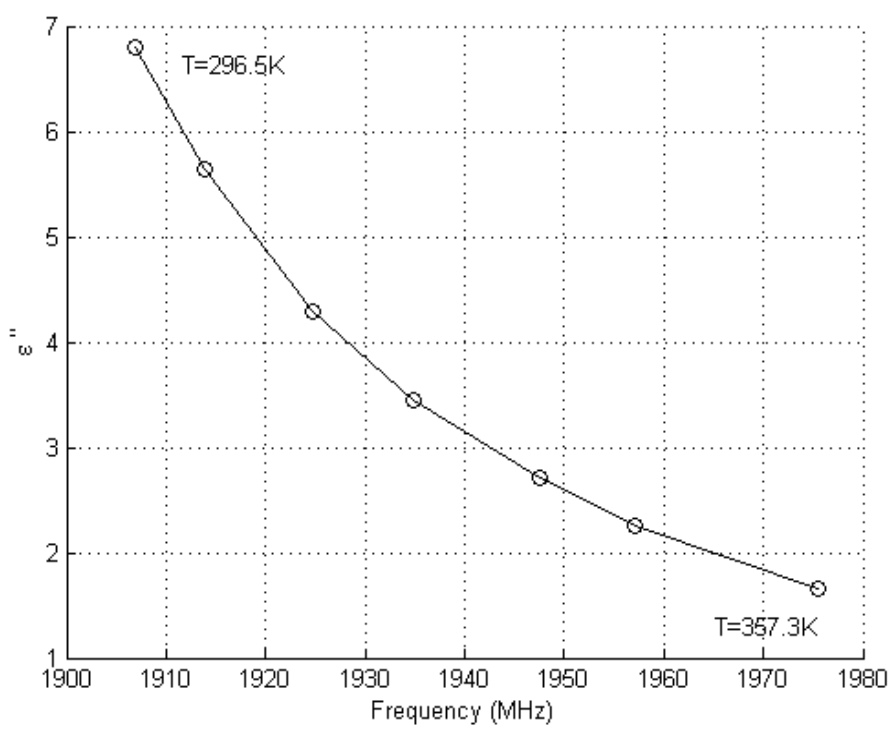

Fig. 3. Measured loss factor of distilled water at different temperatures. 


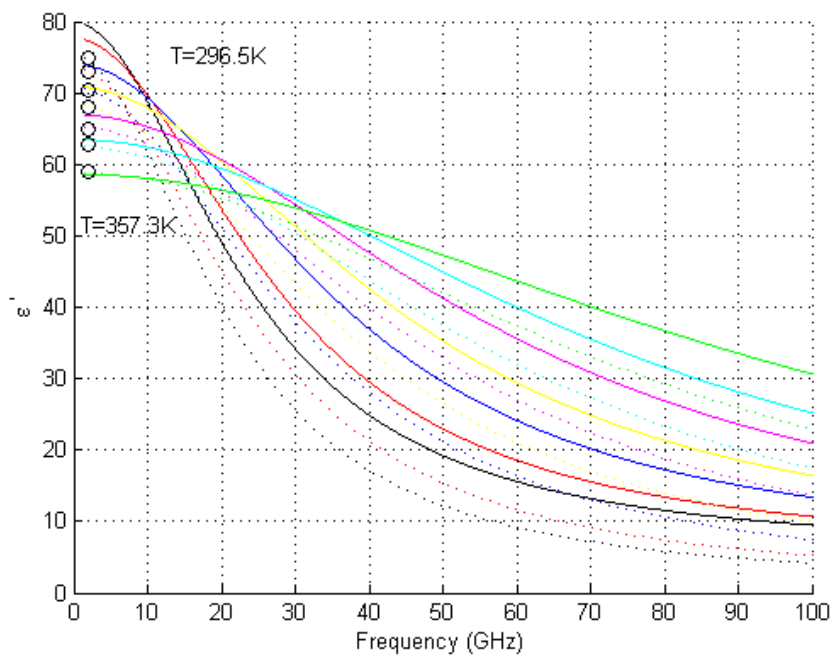

Fig. 4. Results for the dielectric constant of distilled water at different temperatures (Continuos line: model in [1], discontinuous line: proposed method, o: measurements).

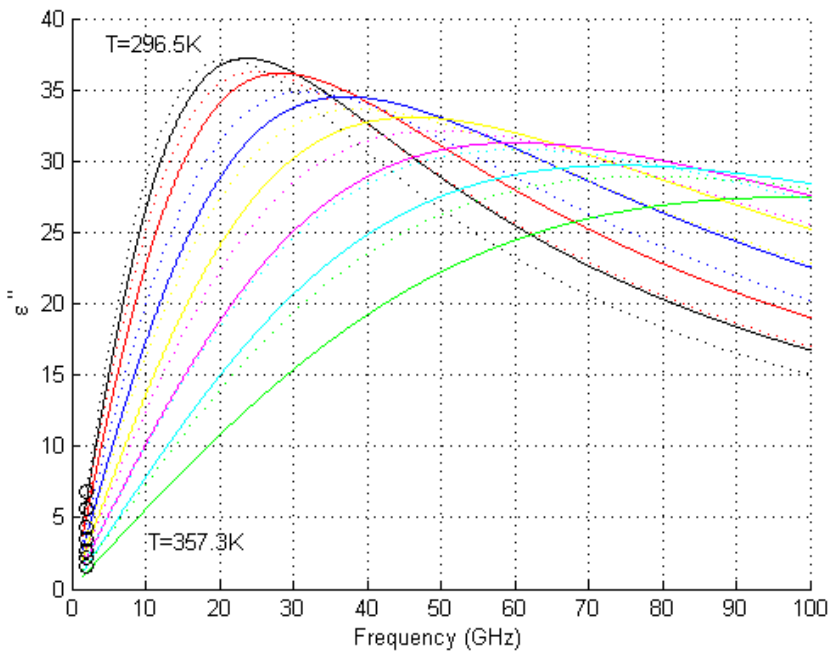

Fig. 5. Results for the loss factor of distilled water at different temperatures. (Continuos line: model in [1], discontinuous line: proposed method, o: measurements). 


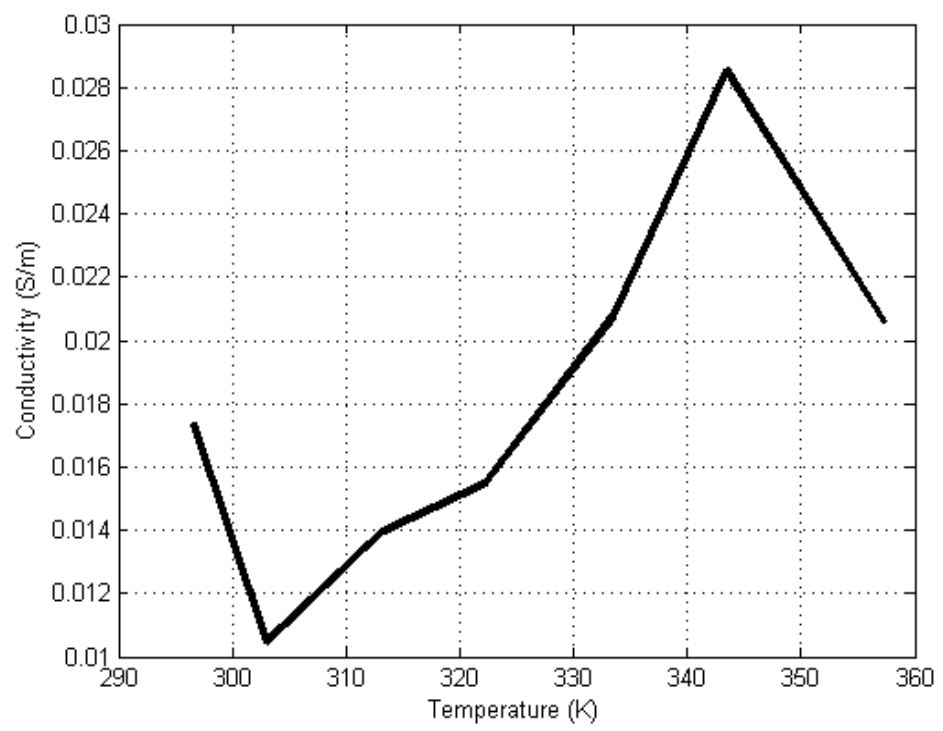

Fig. 6. Conductivity for the distilled water at different temperatures.

However uncertainty for the parameters related with the relaxation time is high, as it could be expected, since the measurement frequencies are far from the frequency value related with the relaxation time using the relationship

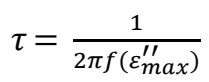

This time value (inverse of the angular frequency at which the loss factor is maximum) leads to a frequency around $20 \mathrm{GHz}$ at room temperature for the distilled water, far from measured values around $1.9 \mathrm{GHz}$.

\section{Conclusions}

In this work we have studied the idea of obtaining the permittivity of a material at different temperatures from measurements using a resonant cavity in a certain wider bandwidth. Since known models such as the Debye are available it is possible to provide an estimation of the permittivity in a broader bandwidth using the cited measurements, the permittivity model and an optimization technique. Genetic algorithms have been used successfully for this purpose. However, care must be taken since selecting wider lower and upper optimization limits may lead to unexpected solutions. As it could have been expected results for the provided solution are worse for farer frequencies from the measurement one. The main uncertainty becomes for the relaxation time since, at the measured frequencies, different combinations for this parameter may lead to reasonable values for the fitness function and more work is envisaged in this direction. 


\section{References}

1. Álvarez, A., Fayos-Fernández, J., Monzó-Cabrera, J., Cocero, M.J. and Mato R.B. (2017), Journal of Food Engeeneering, 197, 98-106.

2. Debye, P. (1929) Chemical Catalogue Company, New York.

3. Tofighi, M. R. (2009), IEEE Transactions on Microwave Theory and Techniques, 17, 2588-2596.

4. Lozano-Guerrero, A.J., Monzó-Cabrera, J., Pedreño-Molina, J.L., ArtésHernández, F., Díaz-Morcillo, A. (2016), Global Congress on Microwave Energy Applications, Cartagena, (SPAIN). 